\title{
Graphene-Assisted Goos-Hänchen Shift in a Planar Multilayer Configuration in the Visible Light Range
}

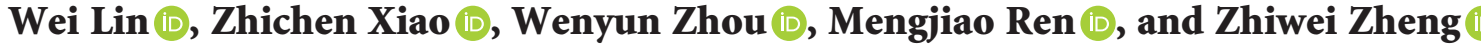 \\ School of Physics and Electronics, Hunan Normal University, Changsha 410081, China \\ Correspondence should be addressed to Zhiwei Zheng; zhzhengzhiwei@163.com
}

Received 26 August 2020; Revised 15 October 2020; Accepted 3 December 2020; Published 15 December 2020

Academic Editor: Shuiqin Zheng

Copyright (c) 2020 Wei Lin et al. This is an open access article distributed under the Creative Commons Attribution License, which permits unrestricted use, distribution, and reproduction in any medium, provided the original work is properly cited.

\begin{abstract}
In this paper, the graphene-assisted Goos-Hänchen $(\mathrm{GH})$ shift of the optical beam reflected from a planar multilayer configuration is investigated. The increased positive Goos-Hänchen shifts can be modulated by adjusting the Fermi energy due to graphene with unique optical properties in the visible light range. Moreover, the GH shift can be tuned by varying the layers of graphene, the thickness of the medium, incident wavelength, and so on. These results will be useful for designing the novel graphene-based optical sensing and switching.
\end{abstract}

\section{Introduction}

When a light beam is completely reflected across two different media, there is a tiny transverse displacement between the practical reflects light and geometric reflects light in the plane of incidence [1]. Also, this displacement has been named as the Goos-Hänchen shift, which was discovered firstly by Goos and Hänchen in 1947 [1], and then, it was addressed by Artmann in 1948 [2]. Owing to its broad application prospects in the field of optical resonance sensor [3], optical temperature sensor [4], optical displacement sensor [5], optical waveguide switch [6], and so on, the GH shift has attracted the attention of the researchers over the past years. However, the value of GH shift is usually small in the optical frequency band. So, the ways of the enhancement of the GH shift are extensively exploited in various structures including photonic crystal [7], metamaterials [8], metalcladding waveguides [9], and multilayer structure [10]. However, the new method of flexible and effective regulation and enhancement of the GH shift needs further research.

Recently, graphene, as a new two-dimensional carbon material, has been useful to enhance the GH shift for satisfying the practical application [11-15]. In addition, the charge carrier concentration and photoconductivity of graphene can be flexibly adjusted by adding bias voltage or chemical modification to produce excellent optical properties such as fast response, broadband, and ultrahigh electron mobility [16-20]. The GH shift was initially defined on the basis of the frustrated total internal reflection (FTIR), which means that the decay wave will transfer energy to the second medium, if the third medium with a high index of refraction is placed within a few wavelengths from the interface between the first and second medium. The reflected beam and transmitted beam in the process of blocked total internal reflection showed that the transmitted beam has the same GH shift as the reflected beam in the symmetric structure $[21,22]$. Due to the characteristics of quantum tunneling in FTIR, light is coupled into the second prism with a symmetric biprism structure with low transmission amplitude, which significantly reproduces the GH shift. The graphene-assisted Goos-Hänchen shift in a two-prism frustrated total internal reflection configuration is investigated, and the GH shift can be enhanced by transmission resonance to more than 10 times the wavelength order, which could be used in the design of optical sensors and optical switches [23]. Martinez and Jalil found that, in the case of TM polarization, the charge and current may have opposite effects on the GH shift [24]. In addition, it is found that the GH shift can be directly controlled by changing electrical or chemical modification of the charge carrier density of graphene. Moreover, due to the transmission resonance relevant in low-terahertz frequency domain, the 
GH shift can be significantly enhanced by the graphene sheet. Above all, electrical tunability of the GH shift from the graphene-on-dielectric surface offers a new possibility to optical devices in information processing and plays a vital role in the development of detections.

In this paper, we study the GH shift in the visible band based on graphene-boron nitride- (BN-) $\mathrm{SiO}_{2}$ heterostructures. To facilitate the analysis, we only consider the transverse magnetic (TM) wave. The composite structure can effectively enhance the GH shift of the visible band. Also, we can realize the control of displacement by adjusting the Fermi energy of graphene. Besides, the GH shift is also related to the layers of graphene, and the thickness of $\mathrm{BN}$ and $\mathrm{SiO}_{2}$. The results based on the proposed structure will provide a new way for developing optical sensing and optical switches.

\section{Proposed Structure and the Simulation Method}

In the graphene- $\mathrm{BN}-\mathrm{SiO}_{2}$-based planar multilayer structure, the GH shift diagram of the system is as shown in Figure 1. The hybrid structure is composed of optical glass BF11 (refractive index is $n_{\mathrm{BF}}=1.61$ ), silicon dioxide (refractive index is $n_{\mathrm{SiO}_{2}}=1.457$ ), boron nitride (refractive index is $n_{\mathrm{BN}}=2.1$ ), and graphene, respectively. In this planar structure, the incident light is injected into the left structure under certain conditions, and the total reflection occurs at the $\mathrm{BF}-\mathrm{SiO}_{2}$ interface. The evanescent experienced a brief overflow and passed through the silica layer to the $\mathrm{BN}$ waveguide. The spilt evanescent is amplified at $\mathrm{BN}$ and extends further to the right of the structure. In fact, we can introduce doping by adding transparent electrodes, and the transmission can be continuously adjusted between these two extremes by changing the doping level. When migrating from highly doped graphene to undoped graphene, the decrease in transmittance is caused by absorption and reflection because local changes in the response of the carbon layer may cause deviations from resonance tunneling conditions. In order to simplify the theoretical model, only the TM-polarized incident light is considered in this paper. Graphene surface conductivity can be described in terms of Kubo. Graphene conductivity $\sigma$ can be expressed as $\sigma=\sigma_{\text {intra }}+\sigma_{\text {inter }}$, where $\sigma_{\text {intra }}$ and $\sigma_{\text {inter }}$ are intraband conductivity and interband conductivity, respectively. They are denoted by [25]

$$
\begin{aligned}
\sigma_{\text {intra }} & =\frac{i e^{2} k_{B} T}{\pi \hbar^{2}\left(\omega+i / \tau_{g}\right)}\left\{\frac{E_{f}}{k_{B} T}+2 \ln \left[\exp \left(-\frac{E_{f}}{k_{B} T}\right)+1\right]\right\}, \\
\sigma_{\text {inter }} & =\frac{i e^{2}}{4 \pi \hbar} \ln \left[\frac{2 E_{f}-\left(\omega+i / \tau_{g}\right) \hbar}{2 E_{f}+\left(\omega+i / \tau_{g}\right) \hbar}\right],
\end{aligned}
$$

where $E_{f}$ is the Fermi energy of graphene, $k_{B}$ is the Boltzmann constant, $T$ is kelvin temperature, $e$ and $\hbar$ are the charge constant of the electron and the Planck constant. $\tau$ is electron relaxation time, satisfying $\tau_{g}=\mu E_{f} / e \nu_{f}^{2}$, where the

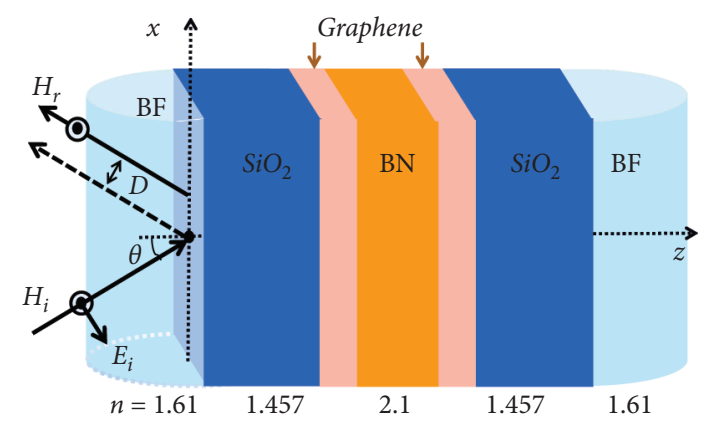

FIgURE 1: Schematic diagram of the GH shift of total reflected beams from a planar multilayer structure.

electron mobility is $\mu=10^{4} \mathrm{~cm}^{2} v^{-1} \mathrm{~s}^{-1}$ and the Fermi velocity is $v_{f}=10^{6} \mathrm{~m} / \mathrm{s}$.

The relative dielectric constant of $\mathrm{BN}$ changes little in the visible band, so we take it as a fixed value in order to simplify the theoretical model. Also, the transverse component of the $\mathrm{BN}$ relative dielectric constant is $u_{x}=4.85$, and the longitudinal component is $u_{z}=2.95$.

The transmission matrix can be used to study the transmission characteristics of light in planar multilayer structures. Therefore, when the incident light penetrates into the structural surface at an angle $\theta$ along the $z$-axis through the special optical glass BF11, the transmission matrix on the $\mathrm{BF}-\mathrm{SiO}_{2}$ interface is expressed as

$$
D_{\mathrm{BF} \longrightarrow \mathrm{SiO}_{2}}=\frac{1}{2}\left[\begin{array}{cc}
1+\eta & 1-\eta \\
1-\eta & 1+\eta
\end{array}\right],
$$

with the parameter $\eta$ given by $\eta=k_{\mathrm{SiO}_{2}, z} / k_{\mathrm{BF}, z}$. Here, $k_{\mathrm{SiO}_{2}, z}$ is the $z$ component of the wave vector $k_{\mathrm{SiO}_{2}}=\sqrt{\varepsilon_{\mathrm{SiO}_{2}}} \omega / c$, where $\omega$ is the angular frequency and $c$ is the speed of light in vacuum.

Then, we consider the propagation of light in $\mathrm{SiO}_{2}$. The propagation matrix in $\mathrm{SiO}_{2}$ can be obtained as

$$
P_{\mathrm{SiO}_{2}}=\left[\begin{array}{cc}
\exp \left(-i k_{\mathrm{SiO}_{2}, z} d_{\mathrm{SiO}_{2}}\right) & 0 \\
0 & \exp \left(i k_{\mathrm{SiO}_{2}, z} d_{\mathrm{SiO}_{2}}\right)
\end{array}\right],
$$

where $d_{\mathrm{SiO}_{2}}$ is the thickness of $\mathrm{SiO}_{2}$.

The other transmission matrices across an interface and the propagation matrices in other different media have similar forms. We can obtain transfer matrix $D$ at other interfaces and propagation matrix $P$ in other media in the same way. Finally, we can get a $2 \times 2$ transfer matrix $M$ related to the two sets of field coefficients in this structure through the transmission matrices and the propagation matrices; namely, the total transmission matrix of the graphene-BN-medium planar multilayer composite structure can be expressed as

$$
M=D_{\mathrm{BF} \longrightarrow \mathrm{SiO}_{2}} P_{\mathrm{SiO}_{2}} D_{\mathrm{SiO}_{2}} \longrightarrow \mathrm{BN}_{\mathrm{BN}} D_{\mathrm{BN}} \longrightarrow \mathrm{SiO}_{2} P_{\mathrm{SiO}_{2}} D_{\mathrm{SiO}_{2}} \longrightarrow \mathrm{BF} \cdot
$$

Based on the transmission matrix, the reflection coefficient of the composite structure can be calculated as 


$$
r(\theta, \omega)=|r(\theta, \omega)| \exp [i \phi(\theta, \omega)],
$$

where $\phi(\theta, \omega)$ is the phase of the reflection coefficient.

The GH shift of the structure is calculated by steady-state phase theory, and its lateral shift is expressed as

$$
D=-\frac{\lambda}{2 \pi} \frac{d \phi(\theta, \omega)}{d \theta}
$$

where $\lambda$ is the incident wavelength.

\section{Results and Discussion}

First of all, we discuss the relationship between the reflectance, reflected phase, and GH shift with different Fermi levels at incident wavelengths of $680 \mathrm{~nm}$, as in Figure 2. The experimental results show that the reflectivity and reflection phase of the structure have a strong correlation with the Fermi energy. In this experiment, we control the Fermi energy level of graphene by doping graphene to different degrees. As the Fermi level increases, the lowest point of reflectivity becomes closer to zero, as shown in Figure 2(a). Then, a significant jump in the reflected phase at the reflected dip is appeared in Figure 2(b), and it can be seen that the larger the Fermi energy level is, the faster the reflected phase dip will be obtained. Moreover, as the phase is monotonically increasing, the GH shift generated is in the negative direction, as shown in Figure 2(c). When Fermi energy $E_{f}$ reaches $1.00 \mathrm{eV}$, the GH shift reaches $-90 \lambda$, but the GH shift only reaches $-12 \lambda$ when $E_{f}=0.92 \mathrm{eV}$. That is because, when the doping degree of the carbon layer increases, the effect of the resonance tunneling condition of the carbon layer increases gradually, so it affects the energy transfer (enhancement) from both absorption and reflection. From the experimental data, in other words, the increase of the Fermi energy level of graphene increases the slope and reflectivity of the reflection phase shift, which leads to the increase of the $\mathrm{GH}$ shift. At the same time, when no graphene material is used, the phase curve decreases monotonically and results in a small shift. This special phenomenon indicates that the positive and negative direction of GH displacement can be easily converted by adjusting the Fermi energy. In general, the enhancement of the Fermi energy level can improve the resonant tunneling effect of the structure, and we can realize the continuous tuning of the GH shift by improving the doping level of graphene.

On the other hand, the relationship between GH displacement and relaxation times is worthwhile to discuss. The change of conductivity will affect the reflection coefficient of the structure and further affect the reflection phase and GH shift of the structure. Figure 3 shows the relationship between the reflection phase, GH shift, and incident angle under different relaxation times. As can be seen from Figure 3(b), with the increase of relaxation time, the GH shift at the resonance angle increases continuously. When $\tau=1.0 \mathrm{ps}$, the $\mathrm{GH}$ shift at the resonance angle reaches $-436 \lambda$, and the enhancement effect of the GH shift is very significant. When the relaxation time decreases, the absolute peak values of transmissivity and the GH shift decrease, which leads to energy loss to some extent. When $\tau^{-1}=0$, that is, the relaxation time approaches infinity, the GH shift can change from negative to positive, indicating that the change of graphene relaxation time can reverse the monotonicity of the reflected phase. In addition, the change of relaxation time does not cause the transverse shift of the reflected angle corresponding to the GH shift peak, that is, the magnitude of resonance angle does not change.

Figure 4 analyzes the influence of the number of graphene layers on the GH shift in this structure. In this set of experiments, we keep the Fermi energy level $\left(E_{f}=1.00 \mathrm{eV}\right)$ and electron relaxation time ( $\tau=0.2 p s)$ of graphene constant and only change the number of graphene layers, that is, change the transfer matrix series. We know that when the number of graphene layers is very small $(N<6)$, graphene conductivity can be expressed in terms of $N \sigma$, where $N$ is the number of graphene layers. According to the previous research, increasing the number of graphene layers can increase the real and imaginary parts of the reflectivity, increase the reflectivity, and decrease the slope of the reflection phase correspondingly. It can be seen from Figure 4 that the GH shift can reach $-90 \lambda$ in the single-layer graphene state, while the double-layer graphene rapidly attenuates to $-40 \lambda$. Also, the critical angle has plummeted from $63.45^{\circ}$ to $63.52^{\circ}$ Therefore, it can be concluded that the number of graphene layers is an important factor affecting GH shift, and single-layer graphene enhances GH displacement significantly. In order to unify the parameters, other studies in this paper only consider the monolayer graphene $(N=1)$.

The thickness of silica cushion and $\mathrm{BN}$ also has an impact on the GH shift, as shown in Figure 5. It can be seen from Figure 5(a) that the absolute value of the GH shift increases gradually with the increase of silica thickness, and the corresponding resonance angle moves to the direction. In this experiment, the tunneling time and consumption of the decay wave are affected by changing the thickness of the medium, so as to realize the influence on the GH shift. The experimental results show that when the thickness of silicon dioxide is fixed and only the thickness of $\mathrm{BN}$ is changed, the observed phenomenon is just the opposite. With the increase of BN thickness, the GH shift gradually decreases. So, we find that the thickness of silica is $675 \mathrm{~nm}$ and the thickness of $\mathrm{BN}$ is $5 \mathrm{~nm}\left(d_{\mathrm{SiO}_{2}}=678 \mathrm{~nm}\right.$ and $d_{\mathrm{BN}}=5 \mathrm{~nm}$ ), and the $\mathrm{GH}$ shift is relatively large and can reach $-90 \lambda$.

The GH shift can be enhanced and adjusted by changing the number of graphene layers, relaxation time, thickness of the medium structure, and so on. However, in practical application, once these structural parameters are determined, it is difficult to change again. In other words, it is impossible to achieve effective dynamic adjustment of optical properties. As can be seen from Figure 6, with the decrease of incident light wave length, the slope of the reflected phase gradually decreases, the absolute value of the GH shift sharply decreases, and the resonance angle of the GH shift blueshifts. According to Figure 6, when the wavelength of incident light is $780 \mathrm{~nm}$, the $\mathrm{GH}$ shift is as long as $-120 \lambda$. But, when the wavelength is reduced to $630 \mathrm{~nm}$, the GH shift is only $-19 \lambda$. 


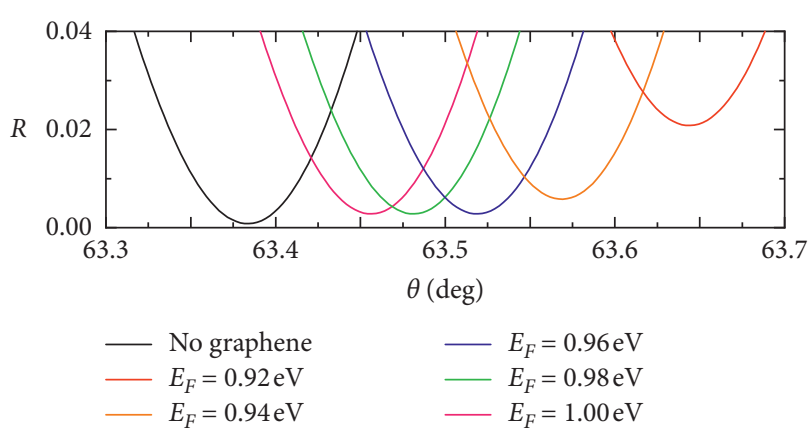

(a)
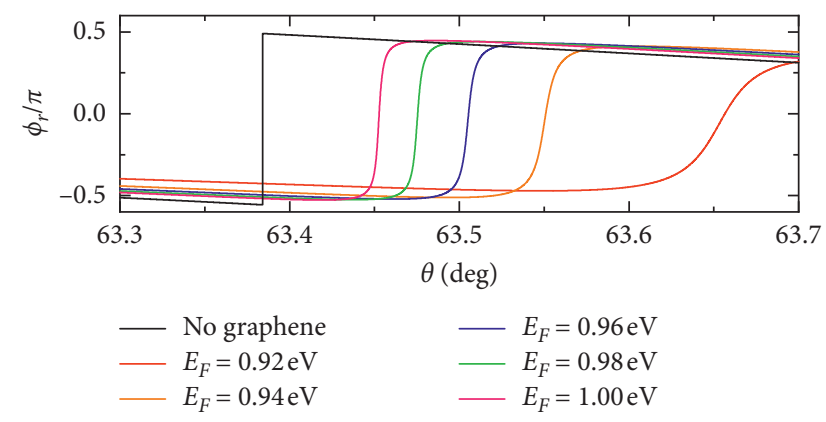

(b)

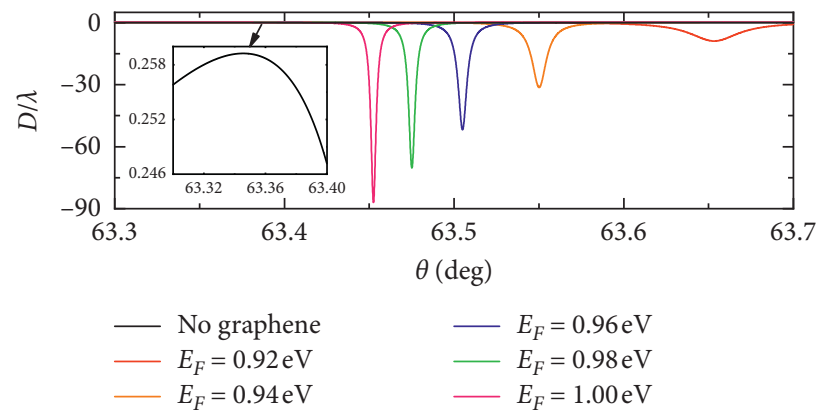

(c)

Figure 2: The dependence of the reflectivity (a), reflection phase (b), and GH shift (c) on the incident angle at different Fermi energies, respectively.

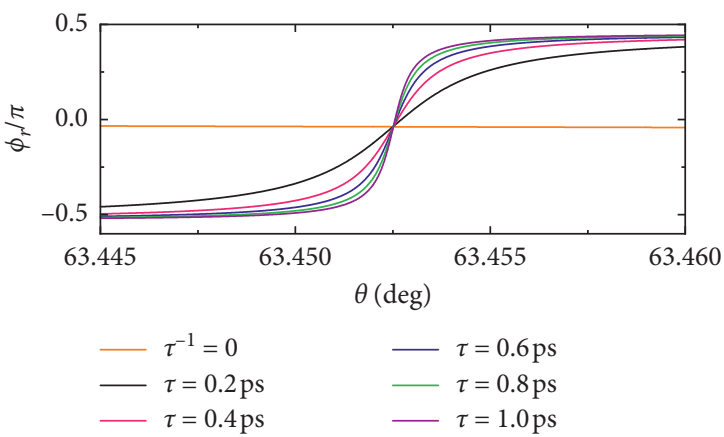

(a)

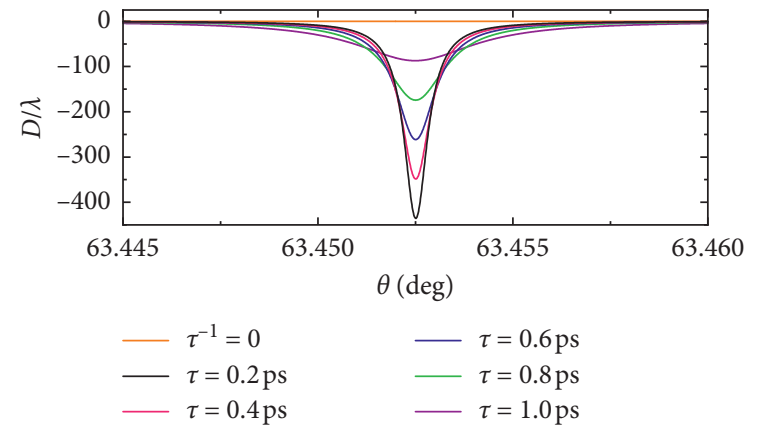

(b)

FIGURE 3: Relationship between the reflection phase, GH shift, and incident angle at different relaxation times.

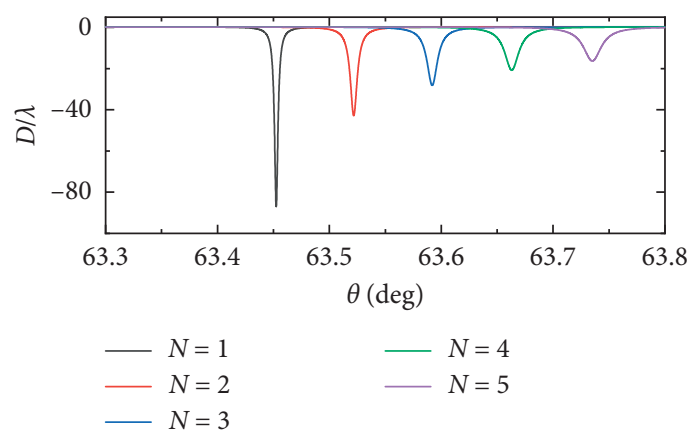

FIGURE 4: Relationship between the GH shift and incident angle at different number of layers of graphene. 


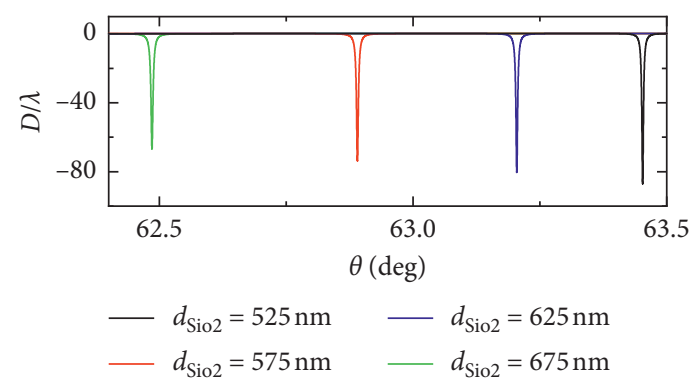

(a)

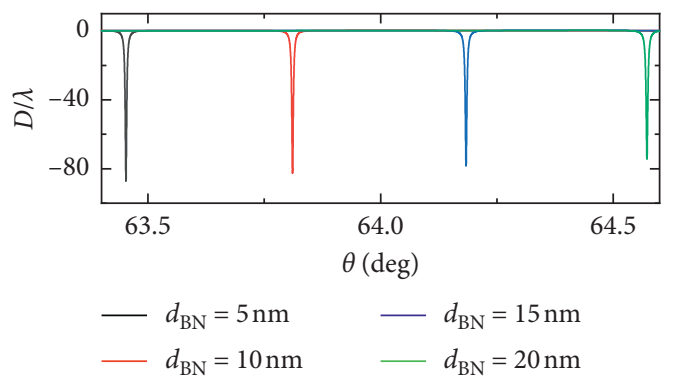

(b)

Figure 5: The variation of the $\mathrm{GH}$ shift with the incident angle at different thicknesses, while (a) changing the thickness of $\mathrm{SiO}_{2}$ $\left(d_{\mathrm{BN}}=5 \mathrm{~nm}\right)$ and $(\mathrm{b})$ changing the thickness of $\mathrm{BN}\left(d_{\mathrm{SiO}_{2}}=675 \mathrm{~nm}\right)$.

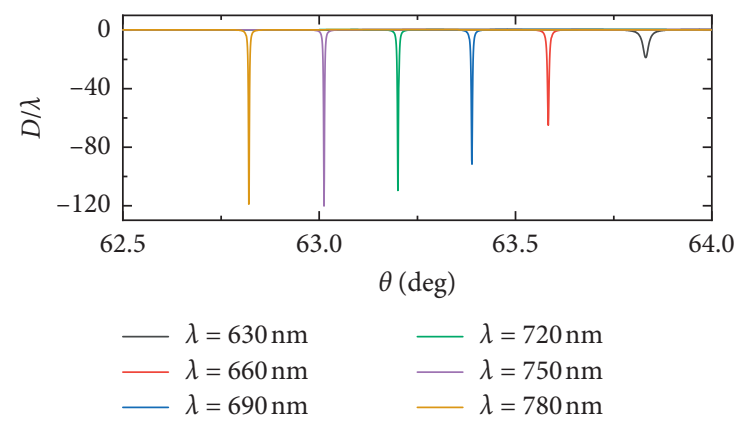

FIgURE 6: Relationship between the GH shift and incident angle at different wavelengths.

\section{Conclusions}

In summary, this paper mainly explores the GH shift of reflected light in the visible range, based on the graphene$\mathrm{BN}-\mathrm{SiO}_{2}$ composite structure which is proposed. We have found that the $\mathrm{GH}$ shift can be enhanced by adjusting the Fermi level of grapheme and the number of the graphene layer. Furthermore, we discover that the GH shift can be changed by adjusting the thickness of some materials in this structure, such as silicon dioxide layer and boron nitride layer. Also, we believe that the result will help develop the potential applications in optical sensing and optical switches.

\section{Data Availability}

Figures 2-6 data used to support the findings of this study are available from the corresponding author upon request.

\section{Conflicts of Interest}

The authors declare that there are no conflicts of interest regarding the publication of this paper.

\section{Acknowledgments}

This work was partially supported by the National Natural Science Foundation of China (Grant no. 61905076), the Hunan Provincial Natural Science Foundation of China (Grant no. 2019JJ50373), Innovation and Entrepreneurship Training Program for College Students in Hunan Province
(Grant no. 201910542053), and the College Students Innovations Project of Hunan Province (Grant no. 2020RC1003).

\section{References}

[1] F. Goos and H. Hänchen, "Ein neuer und fundamentaler versuch zur totalreflexion," Annalen der Physik, vol. 436, no. 7-8, pp. 333-346, 1947.

[2] K. Artmann, "Berechnung der seitenversetzung des totalreflektierten strahles," Annalen der Physik, vol. 437, no. 1-2, pp. 87-102, 1948.

[3] X. Yin and L. Hesselink, "Goos-Hänchen shift surface plasmon resonance sensor," Applied Physics Letters, vol. 89, no. 26, Article ID 261108, 2006.

[4] X. Wang, C. Yin, J. Sun et al., "High-sensitivity temperature sensor using the ultrahigh order mode-enhanced GoosHänchen effect," Optics Express, vol. 21, no. 11, pp. 13380-13385, 2013.

[5] T. Yu, H. Li, Z. Cao, Y. Wang, Q. Shen, and Y. He, "Oscillating wave displacement sensor using the enhanced Goos-Hänchen effect in a symmetrical metal-cladding optical waveguide," Optics Letters, vol. 33, no. 9, pp. 1001-1003, 2008.

[6] T. Sakata, H. Togo, and F. Shimokawa, "Reflection-type $2 \times 2$ optical waveguide switch using the Goos-Hänchen shift effect," Applied Physics Letters, vol. 76, no. 20, pp. 2841-2843, 2000.

[7] I. V. Soboleva, V. V. Moskalenko, and A. A. Fedyanin, "Giant Goos-Hänchen effect and fano resonance at photonic crystal surfaces," Physical Review Letters, vol. 108, Article ID 123901, 2012. 
[8] W. J. Yu, H. Sun, and L. Gao, "Enhanced normal-incidence Goos-Hänchen effects induced by magnetic surface plasmons in magneto-optical metamaterials," Optics Express, vol. 26, no. 4, pp. 3956-3973, 2018.

[9] L. Chen, Z. Cao, F. Ou, H. Li, Q. Shen, and H. Qiao, "Observation of large positive and negative lateral shifts of a reflected beam from symmetrical metal-cladding waveguides," Optics Letters, vol. 32, no. 11, pp. 1432-1434, 2007.

[10] Y. Hirai, K. Matsunaga, Y. Neo, T. Matsumoto, and M. Tomita, "Observation of Goos-Hänchen shift in plasmoninduced transparency," Applied Physics Letters, vol. 112, no. 5, Article ID 051101, 2018

[11] A. K. Geim and K. S. Novoselov, "The rise of graphene," Nature Materials, vol. 6, no. 3, pp. 183-191, 2007.

[12] Z. Zheng, C. Zhao, S. Lu et al., "Microwave and optical saturable absorption in graphene," Optics Express, vol. 20, no. 21, pp. 23201-23214, 2012.

[13] Y. Chen, G. Jiang, S. Chen et al., "Mechanically exfoliated black phosphorus as a new saturable absorber for both Q-switching and mode-locking laser operation," Optics Express, vol. 23, no. 10, pp. 12823-12833, 2015.

[14] S. Chen, L. Miao, X. Chen et al., "Few-layer topological insulator for all-optical signal processing using the nonlinear kerr effect," Advanced Optical Materials, vol. 3, no. 12, pp. 1769-1778, 2015.

[15] M. Cheng, P. Fu, X. Chen, X. Zeng, S. Feng, and R. Chen, "Giant and tunable Goos-Hanchen shifts for attenuated total reflection structure containing graphene," Journal of the Optical Society of America B, vol. 31, no. 10, pp. 2325-2329, 2014.

[16] J. Guo, L. Wu, X. Dai et al., "Absorption enhancement and total absorption in a graphene-waveguide hybrid structure," AIP Advances, vol. 7, no. 2, Article ID 025101, 2017.

[17] L. Jiang, Q. Wang, Y. Xiang, X. Dai, and S. Wen, "Electrically tunable Goos-Hanchen shift of light beam reflected from a graphene-on-dielectric surface," IEEE Photonics Journal, vol. 5, no. 3, Article ID 6500108, 2013.

[18] Q. You, Y. Shan, S. Gan, Y. Zhao, X. Dai, and Y. Xiang, "Giant and controllable Goos-Hänchen shifts based on surface plasmon resonance with graphene-MoS2 heterostructure," Optical Materials Express, vol. 8, no. 10, pp. 3036-3048, 2018.

[19] X. Zhou, S. Liu, Y. Ding, L. Min, and Z. Luo, "Precise control of positive and negative Goos-Hänchen shifts in graphene," Carbon, vol. 149, pp. 604-608, 2019.

[20] R. Yu, V. Pruneri, and F. J. García de Abajo, "Resonant visible light modulation with graphene," ACS Photonics, vol. 2, no. 4, pp. 550-558, 2015.

[21] S. R. Seshadri, "Goos-Hänchen beam shift at total internal reflection," Journal of the Optical Society of America A, vol. 5, no. 4, pp. 583-585, 1988.

[22] A. A. Bocharov, "Goos-Hänchen shift of a transmitted light beam in frustrated total internal reflection for moderately large gap widths," Optics Communications, vol. 389, pp. 297-302, 2017.

[23] Y. Chen, Y. Ban, Q.-B. Zhu, and X. Chen, "Graphene-assisted resonant transmission and enhanced Goos-Hänchen shift in a frustrated total internal reflection configuration," Optics Letters, vol. 41, no. 19, pp. 4468-4471, 2016.

[24] J. C. Martinez and M. B. A. Jalil, "Theory of giant Faraday rotation and Goos-Hänchen shift in graphene," Europhysics Letters, vol. 96, no. 2, Article ID 27008, 2011.

[25] P.-Y. Chen and A. Alù, "Atomically thin surface cloak using graphene monolayers,” ACS Nano, vol. 5, no. 7, pp. 5855-5863, 2011. 